International Mathematical Forum, 2, 2007, no. 16, 753 - 764

\title{
The Filtering Problem in Duals of Nuclear Fréchet Spaces
}

\author{
Deng DING \\ Department of Mathematics \\ Faculty of Science and Technology \\ University of Macau, Macao, China \\ dding@umac.mo
}

\begin{abstract}
The filtering problem, on which the signal process and observation process are both diffusions in dual of nuclear Fréchet space, is considered. Martingale representation theorem and Girsanov's theorem in such space are proved. The innovation process is discussed. The filtering equation for the best estimate of signal process on observation process, is established by the innovation approach.
\end{abstract}

Mathematics Subject Classifications: 60H20, 60H30, 65H99

Keywords: Filtering problem, Nuclear space, Diffusions, Innovation process, Stochastic evolution equation, Filtering equation

\section{Introduction}

The filtering problem consists in estimating the state of a stochastic differential system from noisy observations. Let $\Phi$ be a separable nuclear Fréchet space with the strong topological dual $\Phi^{\prime}$. In this paper, we consider the following stochastic system: the state process (signal process) $X_{t}$ is a $\Phi^{\prime}$-valued diffusion process given by a stochastic evolution equation (SEE):

$$
\mathrm{d} X_{t}=A X_{t} \mathrm{~d} t+F(t) \mathrm{d} W_{t}, \quad t \geq 0,
$$

and the observation process $Y_{t}$ is another $\Phi^{\prime}$-valued diffusion process given by a stochastic differential equation (SDE):

$$
\mathrm{d} Y_{t}=H\left(X_{t}\right) \mathrm{d} t+\mathrm{d} W_{t}, \quad t \geq 0
$$

where $W_{t}$ is a $\Phi^{\prime}$-valued Wiener process, $F(t)$ is an $\mathcal{L}\left(\Phi^{\prime}, \Phi^{\prime}\right)$-valued stochastic process, $A \in \mathcal{L}\left(\Phi^{\prime}, \Phi^{\prime}\right)$ and $H(\cdot)$ is a mapping from $\Phi^{\prime}$ into $\Phi^{\prime}$. Our filtering 
problem is to obtain a mathematical expression, more exactly, a stochastic differential equation, for the best estimate $\widehat{X}_{t}$ of the state $X_{t}$ based on the observation $Y_{t}$. Our main idea lies on the innovation approach which was first used to the nonlinear filtering problem by Kunita et al in [3], and was extended to the Hilbert space valued diffusion processes by Korezioglu in [5], to the distribution valued processes by Korezioglu and Martias in [6].

The organization of the paper is as follows. After this introduce we collect some necessary notations of nuclear spaces as well as some useful results on $\Phi^{\prime}$ valued diffusion processes, which can be found in [2], in Section 2. In Section 3, we prove the martingale representation theorems for real valued and $\Phi^{\prime}$-valued martingales, respectively, and establish a Girsanov's theorem for the $\Phi^{\prime}$-valued diffusion $Y_{t}$. In Section 4, we deal with the innovation process corresponding to our filtering problem and show the representation theorems for this innovation process. In Section 5, we show the main result of this paper: the best estimate $\widehat{X}_{t}=\mathbf{E}\left[X_{t} \mid \mathcal{G}_{t}\right]$, where $\mathcal{G}_{t}$ is the $\sigma$-algebra generated by the observation $\left\{Y_{s}: 0 \leq s \leq t\right\}$, satisfies a stochastic evolution equation on the space $\Phi^{\prime}$, which is called the filtering equation.

\section{Preliminaries}

We recall a locally convex space $\Psi$ is nuclear if there exists a convex circled neighborhood base $\mathcal{U}_{h}(\Psi)$ such that, for every $U \in \mathcal{U}_{h}(\Psi), \Psi(U)$ is a Hilbert space and the canonical mapping $k_{U}: \Psi \rightarrow \Psi(U)$ is nuclear, where $\Psi(U)$ is the completion of quotient space $\Psi / \operatorname{ker}\left(p_{U}\right)$ under the norm $\left\|k_{U} \psi\right\|_{U}=p_{U}(\psi)$, $\psi \in \Psi\left(p_{U}(\cdot)\right.$ is the gauge functional of $\left.U\right)$. For any $U, V \in \mathcal{U}_{h}(\Psi)$ with $U \subset V$, denote $k_{U, V}$ as the canonical mapping from $\Psi(U)$ into $\Psi(V)$ such that $k_{V}=k_{U} k_{U, V}$. It is obvious that $k_{U, V}$ is linear and continuous.

Let $\Psi^{\prime}$ denote the strong topological dual of $\Psi$. Then for every $U \in \mathcal{U}_{h}(\Psi)$, the dual specs of $\Psi(U)$ can be identified by the space $\Psi^{\prime}\left[U^{\circ}\right]$ under the dual operator $i_{U^{\circ}}$ of $k_{U}$, where $U^{\circ}$ is the polar set of $U, \Psi^{\prime}\left[U^{\circ}\right]$ is the completion of the subspace spanned by $U^{\circ}$ under $\Psi^{\prime}$ with the norm $p_{U^{\circ}}$, and $i_{U^{\circ}}$ is the embedding mapping from $\Psi^{\prime}\left[U^{\circ}\right]$ into $\Psi^{\prime}$. About detail definitions and general properties of nuclear spaces, one can refer to [10].

In this paper, we always assume that $\Phi$ be a separable nuclear Fréchet space with the strong topological dual $\Phi^{\prime}$. Then, $\Phi$ is a reflexive, barreled and bornological space, and $\Phi^{\prime}$ is a complete nuclear space $([10,11])$.

Let $(\Omega, \mathcal{F}, \mathbf{P})$ be a complete probability space with a filtration $\left\{\mathcal{F}_{t}\right\}_{t \geq 0}$ which satisfies the usual hypothesis. A set $\left\{X_{t}^{U}: U \in \mathcal{U}_{h}\left(\Phi^{\prime}\right)\right\}$ is said to be a projective system if for every $U \in \mathcal{U}_{h}\left(\Phi^{\prime}\right), X_{t}^{U}$ is a $\Phi^{\prime}(U)$-valued stochastic process, and for any $U, V \in \mathcal{U}_{h}\left(\Phi^{\prime}\right)$ with $U \subset V, k_{V, U} X_{t}^{U}=X_{t}^{V}, P$-a.s.. For every projective system $\left\{X_{t}^{U}: U \in \mathcal{U}_{h}\left(\Phi^{\prime}\right)\right\}$ there exists a unique $\Phi^{\prime}$-valued process $\left\{X_{t}\right\}$, which is called the limit of this projective system, so that for 
every $U \in \mathcal{U}_{h}\left(\Phi^{\prime}\right), k_{U} X_{t}=X_{t}^{U}, \mathbf{P}$-a.s. $([11])$.

Assume $T>0$ is fixed. Let $Q(\cdot, \cdot)$ be a continuous positive definite bilinear form on $\Phi \times \Phi$, and let $W_{t}$ be a $\Phi^{\prime}$-valued Wiener process with the covariance $Q(\cdot, \cdot)$. Then, using the nuclear theorem and the regularization technique ([7]), we can show that there exists a $G \in \mathcal{U}_{h}(\Phi)$ and a $\Phi^{\prime}\left[G^{\circ}\right]$-valued Wiener process $W_{t}^{G}$ with covariance $Q_{G}$, satisfy the following property:

(P1) $i_{G^{\circ}} W_{t}^{G}=W_{t}, 0 \leq t \leq T, P$-a.s., and $Q(\cdot, \cdot)=Q_{G}\left(k_{G}, k_{G} \cdot\right)$.

In the following, we introduce the stochastic integrals with respective to the $\Phi^{\prime}$-valued Wiener process, as well as the $\Phi^{\prime}$-valued diffusions given by SEE (1.1). For the details we refer to [2].

Let $H$ be a separable Hilbert spaces and let $\|\cdot\|_{1}$ be the trace norm. Let $\Lambda_{Q}\left(\Phi^{\prime}, H\right)$ be the class of all $\mathcal{L}\left(\Phi^{\prime}, H\right)$-valued processes $F^{H}(\cdot)$ which satisfies that $F^{H}(\cdot) \xi, \xi \in \Phi^{\prime}$, is an $H$-valued predictable process and

$$
\mathbf{E}\left[\int_{0}^{T}\left\|F^{H}(t) Q F^{H *}(t)\right\|_{1} \mathrm{~d} t\right]<\infty,
$$

where $\mathcal{L}(H, \Phi)$-valued process $F^{H *}(\cdot)$ is the adjoint process of $F^{H}(\cdot)$ given by

$$
\xi\left[F^{H *}(t) h\right]=<h, F^{H}(t) \xi>_{H}, \quad \forall(\xi, h) \in \Phi^{\prime} \times H,
$$

and $Q: \Phi \rightarrow \Phi^{\prime}$ is a symmetric, positive and nuclear operator given by $\left(Q \varphi_{1}\right)\left[\varphi_{2}\right]=Q\left(\varphi_{1}, \varphi_{2}\right), \varphi_{1}, \varphi_{2} \in \Phi$. Then $\Lambda_{Q}\left(\Phi^{\prime}, H\right)$ is a Hilbert space under the inner product:

$$
\left\langle F_{1}^{H}, F_{2}^{H}\right\rangle=\mathbf{E}\left[\int_{0}^{T}\left\|F_{1}^{H}(t) Q F Z_{2}^{H *}(t)\right\|_{1} \mathrm{~d} t\right],
$$

The set of all simple processes is dense in $\Lambda_{Q}\left(\Phi^{\prime}, H\right)$. Hence, there exists a unique, isometric integral, denoted by $\int_{0}^{t} F_{s}^{H} \mathrm{~d} W_{s}$, from $\Lambda_{Q}\left(\Phi^{\prime}, H\right)$ into the space of all $H$-valued continuous square integrable martingales. Moreover, if $G \in \mathcal{U}_{h}(\Phi)$ and $\left\{W_{t}^{G}\right\}$ are given in $(\mathrm{P} 1)$, and $F_{G}^{H}(\cdot)=F^{H}(\cdot) i_{G^{\circ}}$, then

$$
\int_{0}^{t} F^{H}(s) \mathrm{d} W_{s}=\int_{0}^{t} F_{G}^{H}(s) \mathrm{d} W_{s}^{G}, \quad 0 \leq t \leq T .
$$

Let $\Lambda_{Q}\left(\Phi^{\prime}, \Phi^{\prime}\right)$ be the set of all $\mathcal{L}\left(\Phi^{\prime}, \Phi^{\prime}\right)$-valued predictable processes $F(t)$ so that

$$
\mathbf{E}\left[\int_{0}^{T} Q\left(F^{*}(t) \varphi, F^{*}(t) \varphi\right) \mathrm{d} t\right]<\infty, \quad \forall \varphi \in \Phi
$$

Let $F(\cdot) \in \Lambda_{Q}\left(\Phi^{\prime}, \Phi^{\prime}\right)$. Then for each $U \in \mathcal{U}_{h}\left(\Phi^{\prime}\right), F^{U}(\cdot)=k_{U} F(\cdot)$ is an $\mathcal{L}\left(\Phi^{\prime}, \Phi^{\prime}(U)\right)$-valued process such that $F^{U}(\cdot) \in \Lambda_{Q}\left(\Phi^{\prime}, \Phi^{\prime}(U)\right)$. Thus, the integral $\int_{0}^{t} F^{U}(s) \mathrm{d} W_{s}$ exists, and the set $\left\{\int_{0}^{t} F^{U}(s) \mathrm{d} W_{s}: U \in \mathcal{U}_{h}\left(\Phi^{\prime}\right)\right\}$ is a projective system. Define the unique limit of this projective system as the stochastic integral of $F(\cdot)$ and denote as $\int_{0}^{t} F(s) \mathrm{d} W_{s}$. This integral has the properties: 
(P2) $\int_{0}^{t} F(s) \mathrm{d} W_{s}, 0 \leq t \leq T$ is a $\Phi^{\prime}$-valued continuous square integrable martingale.

(P3) For every $U \in \mathcal{U}_{h}\left(\Phi^{\prime}\right)$,

$$
k_{U}\left(\int_{0}^{t} F(s) \mathrm{d} W_{s}\right)=\int_{0}^{t} F^{U}(s) \mathrm{d} W_{s}, \quad 0 \leq t \leq T .
$$

(P4) For every $\varphi \in \Phi$, let $F_{t}(\varphi)$ be an $\mathcal{L}\left(\Phi^{\prime}, R\right)$-valued process defined by $F_{t}(\varphi) \xi=F(t) \xi[\varphi], \xi \in \Phi^{\prime}$, then $F_{t}(\varphi) \in \Lambda_{Q}\left(\Phi^{\prime}, R\right)$ and

$$
\left(\int_{0}^{t} F(s) \mathrm{d} W_{s}\right)[\varphi]=\int_{0}^{t} F_{s}(\varphi) \mathrm{d} W_{s}, \quad 0 \leq t \leq T .
$$

(P5) For any $\varphi_{1}, \varphi_{2} \in \Phi$,

$$
\left\langle\int_{0}^{\cdot} F_{s}\left(\varphi_{1}\right) \mathrm{d} W_{s}, \int_{0}^{\cdot} F_{s}\left(\varphi_{2}\right) d W_{s}\right\rangle_{t}=\int_{0}^{t} Q\left(F^{*}(s) \varphi_{1}, F^{*}(s) \varphi_{2}\right) \mathrm{d} s .
$$

Now we turn to SEE (1.1). Assume the operator $A$ and the process $S^{*}(t)$ satisfy the conditions:

(A1) $A^{*} \in \mathcal{L}(\Phi, \Phi)$, the dual operator of $A$, is an infinitesimal generator of a $\mathcal{L}(\Phi, \Phi)$-operator semigroup $S^{*}(t)$ of class $\left(C_{0}, 1\right)$ with the topology defined by $\mathcal{U}_{h}(\Phi)$

(A2) $F(\cdot) \in \Lambda_{Q}\left(\Phi^{\prime}, \Phi^{\prime}\right)$ and

$$
\mathbf{E}\left[\int_{0}^{T} \int_{0}^{t} Q\left(F_{s}\left(S^{*}(t-s) \varphi\right), F_{s}\left(S^{*}(t-s) \varphi\right)\right) \mathrm{d} s \mathrm{~d} t\right]<\infty
$$

where the $\mathcal{L}\left(\Phi^{\prime}, R\right)$-valued process $\left\{F_{s}\left(S^{*}(t-s) \varphi\right): 0 \leq s \leq t\right\}$ is defined as in (P4) and it is regarded as a $\Phi$-valued process since $\Phi$ is reflexive.

Then for every $X_{0} \in \Phi^{\prime} \operatorname{SEE}(1.1)$ has a weak evolution solution $X_{t}$, which is a $\Phi^{\prime}$-valued diffusion given by

$$
X_{t}=S(t) X_{0}+\int_{0}^{t} S(t-s) F(s) \mathrm{d} W_{s}, \quad 0 \leq t \leq T,
$$

and satisfies $\operatorname{SEE}(1.1)$ in the sense: for each $\varphi \in \Phi$,

$$
X_{t}[\varphi]=X_{0}[\varphi]+\int_{0}^{t} X_{s}\left[A^{*} \varphi\right] \mathrm{d} s+\int_{0}^{t} F_{s}(\varphi) \mathrm{d} W_{s}, \quad 0 \leq t \leq T,
$$

where $S(t)$ is denoted the dual operator of $S^{*}(t)$. Finally, we introduce an Itô's formula for the $\Phi^{\prime}$-valued diffusion $X_{t}$ given by (2.7). For every $f \in C_{b}^{2}(R)$ and each $\varphi \in D\left(A^{*}\right)$, we have

$$
\begin{aligned}
\mathrm{d} f\left(X_{t}[\varphi]\right)= & f^{\prime}\left(X_{t}[\varphi]\right) F_{t}(\varphi) \mathrm{d} W_{s}+\left(f^{\prime}\left(X_{s}[\varphi]\right) X_{s}\left[A^{*} \varphi\right]\right. \\
& \left.+\frac{1}{2} f^{\prime \prime}\left(X_{s}[\varphi]\right) Q\left(F_{s}(\varphi), F_{s}(\varphi)\right)\right) \mathrm{d} s
\end{aligned}
$$




\section{Martingale Representation Theorem and Gir- sanov's Theorem}

Let $\mathcal{F}_{t}^{W}=\sigma\left(\sigma\left\{W_{s}: 0 \leq s \leq t\right\} \cup \mathcal{N}\right)$, for every $t \geq 0$, where $\mathcal{N}$ is the collection of all $P$-null sets. By applying Hahn-Banach extension theorem and using the simple processes approximation we can show the following lemma.

Lemma 3.1 Let $H$ be a Hilbert space. Assume $G \in \mathcal{U}_{h}(\Phi)$ and $Z_{G}^{H}(\cdot)$ is an $\mathcal{L}\left(\Phi^{\prime}\left[G^{\circ}\right], H\right)$-valued, $\left\{\mathcal{F}_{t}^{W}\right\}$-predictable process such that

$$
\mathbf{E}\left[\int_{0}^{T}\left\|Z_{G}^{H}(t) Q_{G} Z_{G}^{H *}(t)\right\|_{1} \mathrm{~d} t\right]<\infty .
$$

Then there exists an $\left\{\mathcal{F}_{t}^{W}\right\}$-predictable process $Z^{H}(\cdot) \in \Lambda_{Q}\left(\Phi^{\prime}, H\right)$ such that $Z^{H}(t) i_{G^{\circ}}=Z_{G}^{H}(t), P$-a.s. for every $0 \leq t \leq T$.

Lemma 3.2 Let $M_{t}$ be a $\Phi^{\prime}$-valued, continuous and square integrable $\left(\mathcal{F}_{t}^{W}\right)$ martingale given by the projective system: $\left\{M_{t}^{U}=\int_{0}^{t} Z^{U}(s) \mathrm{d} W_{s}: U \in \mathcal{U}_{h}\left(\Phi^{\prime}\right)\right\}$. Then there exists an $\left\{\mathcal{F}_{t}^{W}\right\}$-predictable process $Z(\cdot) \in \Lambda_{Q}\left(\Phi^{\prime}, \Phi^{\prime}\right)$ such that $k_{U} Z(\cdot)=Z^{U}(\cdot), P$-a.s. and $M_{t}=\int_{0}^{t} Z(s) d W_{s}, 0 \leq t \leq T$.

Proof Define a mapping $\Gamma: \Phi \rightarrow \Lambda_{Q}\left(\Phi^{\prime}, R\right)$ by $(\Gamma \varphi)(\cdot)=\left(Z^{U}(\cdot)\right)^{*} h$, where $U \in \mathcal{U}_{h}\left(\Phi^{\prime}\right)$ is chosen so that there is a $h \in \Phi\left[U^{\circ}\right]$ such that $i_{U^{\circ}} h=\varphi$. Then it is easy to verify that $\Gamma$ is linear and continuous. Define $Z(\cdot) \xi=\left(\Gamma^{*} \xi\right)(\cdot), \forall \xi \in \Phi^{\prime}$. Then $Z(\cdot) \in \Lambda_{Q}\left(\Phi^{\prime}, \Phi^{\prime}\right)$ and so that $k_{U} Z(\cdot)=Z^{U}(\cdot)$ for each $U \in \mathcal{U}_{h}\left(\Phi^{\prime}\right)$, and so that $M_{t}=\int_{0}^{t} Z(s) d W_{s}, 0 \leq t \leq T$.

The next result is a Brownian martingale representation theorem for $\Phi^{\prime}$ valued martingales.

Theorem 3.3 For every $\Phi^{\prime}$-valued, continuous and square integrable $\left(\mathcal{F}_{t}^{W}\right)$ martingale $M_{t}$ with $M_{0}=0$, there exists an $\mathcal{L}\left(\Phi^{\prime}, \Phi^{\prime}\right)$-valued, $\left(\mathcal{F}_{t}^{W}\right)$-predictable process $Z(\cdot) \in \Lambda_{Q}\left(\Phi^{\prime}, \Phi^{\prime}\right)$ such that $M_{t}=\int_{0}^{t} Z(s) \mathrm{d} W_{s}, 0 \leq t \leq T$.

Proof For each $U \in \mathcal{U}_{h}\left(\Phi^{\prime}\right)$, let $M_{t}^{U}=k_{U} M_{t}, 0 \leq t \leq T$. Then $M_{t}^{U}$ is a $\Phi^{\prime}(U)$-valued, continuous and square integrable $\left\{\mathcal{F}_{t}^{W}\right\}$-martingale. By Brownian martingale representation theorem in Hilbert space $([9])$, there exists an $\mathcal{L}\left(\Phi^{\prime}\left[G^{\circ}\right], \Phi^{\prime}(U)\right)$-valued $\left\{\mathcal{F}_{t}^{W}\right\}$-predictable process $Z_{G}^{U}(t)$ which satisfies $(3.1)$ and $M_{t}^{U}=\int_{0}^{t} Z_{G}^{U}(s) \mathrm{d} W_{s}^{G}, 0 \leq t \leq T$. By Lemma 3.1, there exists an $\left\{\mathcal{F}_{t}^{W}\right\}$ predictable process $Z^{U}(\cdot) \in \Lambda_{Q}\left(\Phi^{\prime}, \Phi^{\prime}(U)\right)$ such that $Z^{U}(t) i_{G^{\circ}}=Z_{G}^{U}(t), P$-a.s., and $M_{t}^{U}=\int_{0}^{t} Z^{U}(s) \mathrm{d} W_{s}, 0 \leq t \leq T$.

It is easy to see that $\left\{M_{t}^{U}: U \in \mathcal{U}_{h}\left(\Phi^{\prime}\right)\right\}$ is a projective system. Hence, by Lemma 3.2 there exists an $\mathcal{L}\left(\Phi^{\prime}, \Phi^{\prime}\right)$-valued, $\left\{\mathcal{F}_{t}^{W}\right\}$-predictable process $Z(\cdot)) \in \Lambda_{Q}\left(\Phi^{\prime}, \Phi^{\prime}\right)$ such that $M_{t}=\int_{0}^{t} Z(s) \mathrm{d} W_{s}, 0 \leq t \leq T$. 
Now we consider Girsanov's' theorem for $\Phi^{\prime}$-valued diffusion. Let $h(\cdot)$ be an $\left\{\mathcal{F}_{t}^{W}\right\}$-predictable process such that $h(\cdot) \in \Lambda_{Q}\left(\Phi^{\prime}, R\right)$. Define a $\Phi^{\prime}$-valued process $H_{t}$ by $H_{t}[\varphi]=Q(h(t), \varphi)$ for all $0 \leq t \leq T$ and $\varphi \in \Phi^{\prime}$, where $h(\cdot)$ is regarded as a $\Phi$-valued process since $\Phi$ is reflexive. Let $Y_{t}$ be a $\Phi^{\prime}$-valued diffusion defined by

$$
Y_{t}=\int_{0}^{t} H_{s} \mathrm{~d} s+W_{t}, \quad 0 \leq t \leq T .
$$

By the typical Girsanov's approach, we will show this process is also a $\Phi^{\prime}$ valued Wiener process under a new probability measure. For any $\varphi \in \Phi$ and $x \in \mathbf{R}$, define a real valued process $\theta_{t}^{\varphi, x}$ by

$$
\theta_{t}^{\varphi, x}=\exp \left\{-\int_{0}^{t}(h(s)+x \varphi) \mathrm{d} W_{s}-\frac{1}{2} \int_{0}^{t} Q(h(s)+x \varphi, h(s)+x \varphi) \mathrm{d} s\right\},
$$

where $x \varphi$ is regarded as a continuous linear functional on $\Phi^{\prime}$.

Lemma 3.4 Suppose the process $h(\cdot)$ satisfies that, for every $x \in \mathbf{R}$,

$$
\mathbf{E}\left[\exp \left\{\frac{1}{2}(1+x) \int_{0}^{T} Q(h(s), h(s)) \mathrm{d} s\right\}\right]<\infty .
$$

Then the real valued process $\theta_{t}^{\varphi, x}$ is a continuous $\left(\left\{\mathcal{F}_{t}^{W}\right\}, \mathbf{P}\right)$-exponential martingale with $\mathbf{E}\left[\theta_{t}^{\varphi, x}\right]=1$ for each $0 \leq t \leq T$.

Proof By Novikov's condition on exponential martingales ([4]) we only need to show

$$
\mathbf{E}\left[\exp \left\{\frac{1}{2} \int_{0}^{t} Q(h(s)+x \varphi, h(s)+x \varphi) \mathrm{d} s\right\}\right]<\infty .
$$

Since $Q$ is a continuous positive bilinear form on $\Phi \times \Phi$, we have the inequality

$$
\begin{aligned}
\int_{0}^{T} Q(h(s)+x \varphi, h(s)+x \varphi) \mathrm{d} s \leq & (1+x) \int_{0}^{T} Q(h(s), h(s)) \mathrm{d} s \\
& +x(1+x) T Q(\varphi, \varphi) .
\end{aligned}
$$

Hence, by the condition (3.3) we get

$$
\begin{aligned}
& \mathbf{E}\left[\exp \left\{\frac{1}{2} \int_{0}^{t} Q(h(s)+x \varphi, h(s)+x \varphi) \mathrm{d} s\right\}\right] \\
\leq & \mathbf{E}\left[\exp \left\{\frac{1}{2}(1+x) \int_{0}^{T} Q(h(s), h(s)) \mathrm{d} s\right\}\right] \exp \left\{\frac{1}{2} x(1+x) T Q(\varphi, \varphi)\right\}<\infty .
\end{aligned}
$$

Thus, the lemma is proved.

Theorem 3.5 (Girsanov's theorem). Let $\theta_{t}=\theta_{t}^{0,0}, 0 \leq t \leq T$, and assume that there exists some $x_{0}>0$ such that for each $\varphi \in \Phi$ and $0 \leq t \leq T$,

$$
\mathbf{E}\left[\exp \left\{x_{0}\left|Y_{t}[\varphi]\right|\right\}\right]<\infty
$$

Then, under (3.3), $Y_{t}$ is a $\Phi^{\prime}$-valued $\left(\left\{\mathcal{F}_{t}^{W}\right\}, \overline{\mathbf{P}}\right)$-Wiener process with the same covariance operator $Q$ as $W_{t}$, where $\overline{\mathbf{P}}$ is a probability on $\left(\Omega, \mathcal{F}_{T}^{W}\right)$, defined by $\mathrm{d} \overline{\mathbf{P}}=\theta_{T} \mathrm{~d} \mathbf{P}$ and being equivalent to $\mathbf{P}$, and so that $\mathrm{d} \bar{P}=\theta_{t} \mathrm{~d} \mathbf{P}$ on $\mathcal{F}_{t}^{W}$, $0 \leq t \leq T$. 
Proof By Lemma 3.4, $\theta_{t}$ is a continuous $\left(\left\{\mathcal{F}_{t}^{W}\right\}, \mathbf{P}\right)$-exponential martingale with $\mathbf{E}\left[\theta_{T}\right]=1$, and hence, $\mathrm{d} \overline{\mathbf{P}}=\theta_{T} \mathrm{~d} \mathbf{P}$ define a probability on $\mathcal{F}_{T}^{W}$. Again by Lemma 3.4 , for every $(\varphi, x) \in \Phi \times \mathbf{R}, \theta_{t}^{\varphi, x}$ is also a continuous $\left(\left\{\mathcal{F}_{t}^{W}\right\}, P\right)$ exponential martingale, and noting that we have

$$
\theta_{t}^{\varphi, x}=\theta_{t} \exp \left\{-x Y_{t}[\varphi]-\frac{1}{2} x^{2} t Q(\varphi, \varphi)\right\}, \quad 0 \leq t \leq T
$$

Thus, for each $0 \leq t \leq T$ and every $B \in \mathcal{F}_{t}$, we get

$$
\begin{aligned}
& \int_{B} \theta_{T} \exp \left\{-x Y_{T}[\varphi]-\frac{1}{2} x^{2} T Q(\varphi, \varphi)\right\} \mathrm{d} \mathbf{P} \\
= & \int_{B} \theta_{t} \exp \left\{-x Y_{t}[\varphi]-\frac{1}{2} x^{2} t Q(\varphi, \varphi)\right\} \mathrm{d} \mathbf{P}
\end{aligned}
$$

by the martingale property of $\theta_{t}^{\varphi, x}$. So we get

$$
\begin{aligned}
& \int_{B} \exp \left\{-x Y_{T}[\varphi]-\frac{1}{2} x^{2} T Q(\varphi, \varphi)\right\} \mathrm{d} \overline{\mathbf{P}} \\
= & \int_{B} \exp \left\{-x Y_{t}[\varphi]-\frac{1}{2} x^{2} t Q(\varphi, \varphi)\right\} \mathrm{d} \overline{\mathbf{P}}
\end{aligned}
$$

by the definition of $\overline{\mathbf{P}}$. This expresses the martingale property of the process

$$
\exp \left\{-x Y_{t}[\varphi]-\frac{1}{2} x^{2} t Q(\varphi, \varphi)\right\}, \quad 0 \leq t \leq T,
$$

under $\overline{\mathbf{P}}$. By (3.4) and according to the exponential formula for martingale $([8]), Y_{t}[\varphi]$, is an $\left(\left\{\mathcal{F}_{t}^{W}\right\}, \overline{\mathbf{P}}\right)$-square integrable martingale, and $\langle Y[\varphi], Y[\varphi]\rangle_{t}=$ $t Q(\varphi, \varphi)$. By the linearity of $Q$, it is easy to verify that for every $\varphi_{1}, \varphi_{2} \in \Phi$, $\left\langle Y\left[\varphi_{1}\right], Y\left[\varphi_{2}\right]\right\rangle_{t}=t Q\left(\varphi_{1}, \varphi_{2}\right)$. This implies that $Y_{t}$ is a $\Phi^{\prime}$-valued $\left(\mathcal{F}_{t}^{W}, \bar{P}\right)$ Wiener process with the covariance operator $Q$.

\section{The Innovation Process}

Let $\mathcal{G}$ be a sub $\sigma$-algebra of $\mathcal{F}$, and let $Z$ be a $\Phi^{\prime}$-valued random variable satisfies that $\mathbf{E}\left[\left\|k_{U} Z\right\|_{U}\right]<\infty$ holds for every $U \in \mathcal{U}_{h}\left(\Phi^{\prime}\right)$. Then the conditional expectation of $Z$ given $\mathcal{G}$, denoted by $\mathbf{E}[Z \mid \mathcal{G}]$, exists and is a $\Phi^{\prime}$-valued random variable satisfying:

$$
\mathbf{E}(Z \mid \mathcal{G})[\varphi]=\mathbf{E}\{Z[\varphi] \mid \mathcal{G}\}, \quad \forall \varphi \in \Phi
$$

Let the observation process $Y_{t}$ be a $\Phi^{\prime}$-valued diffusion given in (1.2), more exactly, given by

$$
Y_{t}=\int_{0}^{t} H\left(X_{s}\right) \mathrm{d} s+W_{t}, \quad 0 \leq t \leq T,
$$

and let $\mathcal{G}_{t}=\sigma\left(\sigma\left\{Y_{s}: 0 \leq s \leq t\right\} \cup \mathcal{N}\right), 0 \leq t \leq T$. Then $\left\{\mathcal{G}_{t}\right\}_{0 \leq t \leq T}$ is a subfiltration such that $\mathcal{G}_{t} \subset \mathcal{F}_{t}^{W}$ for every $t$. In the following, for every $\Phi^{\prime}$-valued process $Z_{t}$, we always denote: $\widehat{Z}_{t}=\mathbf{E}\left[Z_{t} \mid \mathcal{G}_{t}\right]$ if the conditional expectation exists. 
Lemma 4.1 Suppose that the $\Phi^{\prime}$-valued $\left\{\mathcal{F}_{t}^{W}\right\}$-predictable process $Z_{t}$ satisfies the conditions that $\mathbf{E}\left[\left\|Z_{t}^{U}\right\|_{U}\right]<\infty$, for all $U \in \mathcal{U}_{h}\left(\Phi^{\prime}\right)$ and $0 \leq t \leq T$, and $\int_{0}^{T} \mathbf{E}\left[\left|Z_{t}[\varphi]\right|^{2}\right] \mathrm{d} t<\infty$ for each $\varphi \in \Phi$. Then the $\Phi^{\prime}$-valued process $\widehat{Z}_{t}$ is $\left\{\mathcal{G}_{t}\right\}$ predictable and satisfies that $\int_{0}^{T} \mathbf{E}\left[\left|\widehat{Z}_{t}[\varphi]\right|^{2}\right] \mathrm{d} t<\infty$ for each $\varphi \in \Phi$.

The proof of this lemma is standard, and we omit it here. Denote $H_{t}=$ $H\left(X_{t}\right)$ for every $0 \leq t \leq T$. The innovation process $N_{t}$ is a $\Phi^{\prime}$-valued, continuous and $\left\{\mathcal{G}_{t}\right\}$-adapted process defined by

$$
N_{t}=Y_{t}-\int_{0}^{t} \widehat{H}_{s} \mathrm{~d} s, \quad 0 \leq t \leq T .
$$

Theorem 4.2 The innovation process $N_{t}$ is a $\Phi^{\prime}$-valued $\left(\left\{\mathcal{G}_{t}\right\}, \mathbf{P}\right)$-Wiener process with covariance operator $Q$.

Proof By (4.2) and (4.3), for every $\varphi \in \Phi$ and any $0 \leq s<t \leq T$ we get

$$
\mathbf{E}\left[\left(N_{t}-N_{s}\right)[\varphi] \mid \mathcal{G}_{s}\right]=\mathbf{E}\left[\int_{s}^{t}\left(H_{u}-\widehat{H}_{u}\right)[\varphi] \mathrm{d} u \mid \mathcal{G}_{s}\right]+\mathbf{E}\left[\left(W_{t}-W_{s}\right)[\varphi] \mid \mathcal{G}_{s}\right]
$$

But we have that $\mathbf{E}\left[\left(W_{t}-W_{s}\right)[\varphi] \mid \mathcal{G}_{s}\right]=\mathbf{E}\left\{E\left[\left(W_{t}-W_{s}\right)[\varphi] \mid \mathcal{F}_{s}^{W}\right] \mid \mathcal{G}_{s}\right\}=0$, and by using Fubini's theorem, we get

$$
\mathbf{E}\left[\int_{s}^{t}\left(H_{u}-\widehat{H}_{u}\right)[\varphi] \mathrm{d} u \mid \mathcal{G}_{s}\right]=\int_{s}^{t} \mathbf{E}\left[\left(H_{u}-\widehat{H}_{u}\right)[\varphi] \mid \mathcal{G}_{s}\right] \mathrm{d} u=0
$$

Hence, we obtain $\mathbf{E}\left[\left(N_{t}-N_{s}\right)[\varphi] \mid \mathcal{G}_{s}\right]=0,0 \leq t \leq T$, and so $N_{t}$ is a $\Phi^{\prime}$-valued $\left(\left\{\mathcal{G}_{t}\right\}, \mathbf{P}\right)$-martingale. Now again by $(4.2)$ and $(4.3)$ we get

$$
N_{t}[\varphi]=\int_{0}^{t}\left(H_{s}-\widehat{H}_{s}\right)[\varphi] \mathrm{d} s+W_{t}[\varphi], \quad 0 \leq t \leq T, \forall \varphi \in \Phi .
$$

Hence, we obtain $\left\langle N\left[\varphi_{1}\right], N\left[\varphi_{2}\right]\right\rangle_{t}=\left\langle W\left[\varphi_{1}\right], W\left[\varphi_{2}\right]\right\rangle_{t}=t Q\left(\varphi_{1}, \varphi_{2}\right)$ for all $\varphi_{1}, \varphi_{2} \in \Phi$. This implies that the innovation process $N_{t}$ is a $\Phi^{\prime}$-valued $\left(\left\{\mathcal{G}_{t}\right\}, \mathbf{P}\right)$ Wiener process with covariance operator $Q$. $\square$

Using the above theorem, and the representation theorem and Girsanov's theorem in Section 3, we can prove a representation theorem for the innovation process. Assume the mapping $H(\cdot)$ satisfies the condition:

(A3) There a bounded and continuous mapping $h(\cdot): \Phi^{\prime} \rightarrow \Phi$ such that $H(\xi)[\varphi]=Q(h(\xi), \varphi)$, for all $(\xi, \varphi) \in \Phi^{\prime} \times \Phi$.

Theorem 4.3 Assume that $(A 3)$ and conditions of Theorem 3.5 are satisfied. Then, for every $\Phi^{\prime}$-valued, continuous and square integrable $\left(\left\{\mathcal{G}_{t}\right\}, P\right)$ martingale $M_{t}$ with $M_{0}=0$, there exists an $\mathcal{L}\left(\Phi^{\prime}, \Phi^{\prime}\right)$-valued, $\left\{\mathcal{G}_{t}\right\}$-predictable process $Z(\cdot) \in \Lambda_{Q}\left(\Phi^{\prime}, \Phi^{\prime}\right)$ such that

$$
M_{t}=\int_{0}^{t} Z(s) \mathrm{d} N_{s}, \quad 0 \leq t \leq T .
$$


Proof From (4.3) we have $Y_{t}=\int_{0}^{t} \widehat{H}_{s} \mathrm{~d} s+N_{t}$ for all $0 \leq t \leq T$. Since $N_{t}$ is a $\Phi^{\prime}$-valued $\left(\left\{\mathcal{G}_{t}\right\}, \mathbf{P}\right)$-Wiener process with covariance operator $Q$, by Girsanov's theorem $Y_{t}$ is a $\Phi^{\prime}$-valued $\left(\left\{\mathcal{G}_{t}\right\}, \overline{\mathbf{P}}\right)$-Wiener process with covariance operator $Q$, where $\overline{\mathbf{P}}$ is a probability on $\mathcal{G}_{T}$ such that $\mathrm{d} \overline{\mathbf{P}}=\theta_{t} \mathrm{~d} \mathbf{P}$ on $\mathcal{G}_{t}$ for each $0 \leq t \leq T$, and $\theta_{T}$ is given by

$$
\theta_{t}=\exp \left\{-\int_{0}^{t} \widehat{h}(s) \mathrm{d} N_{s}-\frac{1}{2} \int_{0}^{t} Q(\widehat{h}(s), \widehat{h}(s)) \mathrm{d} s\right\}, \quad 0 \leq t \leq T,
$$

where $\widehat{h}(t)=\widehat{h\left(X_{t}\right)}, 0 \leq t \leq T$. Define $\bar{M}_{t}=\left(\theta_{t}\right)^{-1} M_{t}, 0 \leq t \leq T$. Then, it is easy to verify that $\bar{M}_{t}$ is a $\Phi^{\prime}$-valued, continuous and square integrable $\left(\left\{\mathcal{G}_{t}\right\}, \overline{\mathbf{P}}\right)$-martingale with $\bar{M}_{0}=0$. Since $\mathcal{G}_{t}=\sigma\left(\sigma\left\{Y_{s}: 0 \leq s \leq t\right\} \cup \mathcal{N}\right)$ for every $0 \leq t \leq T$, by Theorem 3.3 there exists $\left(\left\{\mathcal{G}_{t}\right\}, \overline{\mathbf{P}}\right)$-predictable process $\bar{Z}(\cdot) \in \Lambda_{Q}\left(\Phi^{\prime}, \Phi^{\prime}\right)$ such that

$$
\bar{M}_{t}=\int_{0}^{t} \bar{Z}(s) \mathrm{d} Y_{s}=\int_{0}^{T} \bar{Z}(s) \mathrm{d} N_{s}+\int_{0}^{T} \bar{Z}(s) \widehat{H}_{s} \mathrm{~d} s, \quad 0 \leq t \leq T
$$

or

$$
\bar{M}_{t}[\varphi]=\int_{0}^{T} \bar{Z}_{s}(\varphi) \mathrm{d} N_{s}+\int_{0}^{T} Q\left(\bar{Z}_{s}(\varphi), \widehat{h}(s)\right) \mathrm{d} s, \quad 0 \leq t \leq T, \forall \varphi \in \Phi
$$

where $Q\left(\bar{Z}_{t}(\varphi), \widehat{h}(t)\right)=\bar{Z}_{t}(\varphi) \widehat{H}_{t}$ for each $0 \leq t \leq T$. Also, we can verify that the process $\theta_{t}$ satisfies the equation:

$$
\theta_{t}=1-\int_{0}^{t} \theta_{s} \widehat{h}(s) \mathrm{d} N_{s}, \quad 0 \leq t \leq T
$$

Thus, for each $\varphi \in \Phi$, by using the integration by parts formula we get

$$
\begin{aligned}
M_{t}[\varphi]= & \theta_{t} \bar{M}_{t}[\varphi]=\int_{0}^{T} \theta_{s} \bar{Z}_{s}(\varphi) \mathrm{d} N_{s}+\int_{0}^{T} \theta_{s} Q\left(\bar{Z}_{s}(\varphi), \widehat{h}_{s}\right) \mathrm{d} s \\
& -\int_{0}^{t} \bar{M}_{s}[\varphi] \theta_{s} \widehat{h}(s) \mathrm{d} N_{s}-\int_{0}^{t} \theta_{s} Q\left(\bar{Z}_{s}(\varphi), \widehat{h}(s)\right) \mathrm{d} s \\
= & \int_{0}^{t} \theta_{s}\left[\bar{Z}_{s}(\varphi)-\bar{M}_{s}[\varphi] \widehat{h}(s)\right] \mathrm{d} N_{s}
\end{aligned}
$$

Now denote $Z(t)=\theta_{t}[\bar{Z}(t)-(\widehat{h} \otimes \bar{M})(t)], 0 \leq t \leq T$, where $(\widehat{h} \otimes \bar{M})(\cdot)$ is an $\mathcal{L}\left(\Phi^{\prime}, \Phi^{\prime}\right)$-valued process defined by $(\widehat{h} \otimes \bar{M})(t) \xi=\xi[\widehat{h}(t)] \bar{M}_{t}$ for each $\xi \in \Phi^{\prime}$ and every $0 \leq t \leq T$. Then we can verify that $Z(\cdot)$ is an $\mathcal{L}\left(\Phi^{\prime}, \Phi^{\prime}\right)$-valued, $\left(\mathcal{G}_{t}\right)$-predictable process such that $Z(\cdot) \in \Lambda_{Q}\left(\Phi^{\prime}, \Phi^{\prime}\right)$ and satisfies (4.4). The theorem is proved, $\square$ 


\section{The Filtering Equation}

In this section we denote $h(t)=h\left(X_{t}\right), 0 \leq t \leq T$, where $h(\cdot)$ is given in (A3). Define an $\mathcal{L}\left(\Phi^{\prime}, \Phi^{\prime}\right)$-valued process $(h \otimes X)(\cdot)$ by $(h \otimes X)(t) \xi=\xi[h(t)] X_{t}$ for all $\xi \in \Phi^{\prime}$ and $0 \leq t \leq T$, and an $\mathcal{L}(\Phi, \Phi)$-valued process $(X \otimes h)(t)$ by $(X \otimes h)(t) \varphi=X_{t}[\varphi] h(t)$ for all $\varphi \in \Phi$ and $0 \leq t \leq T$. Using the Hölder inequality, we can proved the following lemma;

Lemma 5.1 If the processes $h(t)$ and $X_{t}$ satisfy:

$$
\mathbf{E}\left[\int_{0}^{T}(Q(h(s), h(s)))^{2} \mathrm{~d} s\right]<\infty \quad \text { and } \quad \mathbf{E}\left[\int_{0}^{T}\left(X_{s}[\varphi]\right)^{4} \mathrm{~d} s\right]<\infty, \forall \varphi \in \Phi .
$$

Then, the process $(h \otimes X)(\cdot) \in \Lambda_{Q}\left(\Phi^{\prime}, \Phi^{\prime}\right)$ and its adjoint process is given by $(h \otimes X)^{*}(\cdot)=(X \otimes h)(\cdot)$. Moreover, the process $(\widehat{h \otimes X})(\cdot)$ exists, and its adjoint process is given by $(\widehat{h \otimes X})^{*}(\cdot)=(\widehat{X \otimes h})(\cdot)$, where $(\widehat{h \otimes X})(t)=$ $\mathbf{E}\left[(h \otimes X)(t) \mid \mathcal{G}_{t}\right], 0 \leq t \leq T$.

Now we will prove our main result on the best estimate $\widehat{X}_{t}$ of the state process $X_{t}$ based on the observation process $Y_{t}$.

Theorem 5.2 Assume that $(A 1)-(A 3)$, the conditions of Theorem 3.5 and Lemma 5.1 are satisfied, and $Q$ is strictly positive, i.e. $Q(\varphi, \varphi)>0$ for all $\varphi \neq 0$. Then $\widehat{X}_{t}$ satisfies the following SEE:

$$
\mathrm{d} \widehat{X}_{t}=A \widehat{X}_{t} \mathrm{~d} t+(\widehat{F}(t)+(\widehat{h \otimes X})(t)-(\widehat{h} \otimes \widehat{X})(t)) \mathrm{d} N_{t}
$$

with the initial condition $\widehat{X}_{0}=X_{0} \in \Phi^{\prime}$.

Proof For every $\varphi \in \Phi$, we have

$$
X_{t}[\varphi]=X_{0}[\varphi]+\int_{0}^{t} X_{s}\left[A^{*} \varphi\right] \mathrm{d} s+\int_{0}^{t} F_{s}(\varphi) \mathrm{d} W_{s}, \quad 0 \leq t \leq T
$$

and

$$
Y_{t}[\varphi]=\int_{0}^{t} H\left(X_{s}\right)[\varphi] \mathrm{d} s+W_{t}[\varphi], \quad 0 \leq t \leq T .
$$

Thus, by Integration by parts formula $([4])$, we get

$$
\begin{aligned}
X_{t}[\varphi] Y_{t}[\varphi]= & \int_{0}^{t} X_{s}[\varphi] H\left(X_{s}\right)[\varphi] \mathrm{d} s+\int_{0}^{t} Y_{s}[\varphi] X_{s}\left[A^{*} \varphi\right] \mathrm{d} s \\
& +\int_{0}^{t} X_{s}[\varphi] \mathrm{d} W_{s}[\varphi]+\int_{0}^{t} Y_{s}[\varphi] F_{s}(\varphi) \mathrm{d} W_{s}+\int_{0}^{t} Q\left(F^{*}(s) \varphi, \varphi\right) \mathrm{d} s
\end{aligned}
$$

Then, as the real valued stochastic filtering equation ([3]), we can show that there exists a real valued $\left\{\mathcal{G}_{t}\right\}$-square integrable martingale $m_{t}^{\varphi}$ such that

$$
\left.\widehat{X}_{t}[\varphi] Y_{t}[\varphi]=m_{t}^{\varphi}+\int_{0}^{t}\left(\widehat{a}_{s}^{\varphi}+\widehat{b}_{s}^{\varphi}\right) \mathrm{d} s+\int_{0}^{t} Y_{s}[\varphi] \widehat{X}_{s}{ }^{*} \varphi\right] \mathrm{d} s
$$


where $a_{t}^{\varphi}=X_{t}[\varphi] H\left(X_{t}\right)[\varphi]$ and $b_{t}^{\varphi}=Q\left(F^{*}(t) \varphi, \varphi\right), 0 \leq t \leq T$. On the other hand, from (4.3) we have

$$
Y_{t}[\varphi]=\int_{0}^{t} \widehat{H}_{s}[\varphi] \mathrm{d} s+N_{t}[\varphi], \quad 0 \leq t \leq T,
$$

where we again denote $H_{t}=H\left(X_{t}\right), 0 \leq t \leq T$. Let

$$
M_{t}=\widehat{X}_{t}-X_{0}-\int_{0}^{t} A \widehat{X}_{s} \mathrm{~d} s, \quad 0 \leq t \leq T .
$$

Then, since $\mathcal{G}_{t} \subset \mathcal{F}_{t}$, it is easy to verify that $M_{t}$ is a $\Phi^{\prime}$-valued $\left\{\mathcal{G}_{t}\right\}$-martingale. Thus, by Theorem 4.3 , there exists a $\left\{\mathcal{G}_{t}\right\}$-predictable process $Z(\cdot) \in \Lambda_{Q}\left(\Phi^{\prime}, \Phi\right)$ such that $M_{t}=\int_{0}^{t} Z(s) \mathrm{d} N_{s}, 0 \leq t \leq T$. Hence we have

$$
\widehat{X}_{t}=X_{0}+\int_{0}^{t} A \widehat{X}_{s} \mathrm{~d} s+\int_{0}^{t} Z(s) \mathrm{d} N_{s}, \quad 0 \leq t \leq T
$$

or, we get

$$
\widehat{X}_{t}[\varphi]=X_{0}[\varphi]+\int_{0}^{t}\left(A \widehat{X}_{s}\right)[\varphi] \mathrm{d} s+\int_{0}^{t} Z_{s}(\varphi) \mathrm{d} N_{s}
$$

Applying Integration by parts formula to (5.3) and (5.5), we obtain

$$
\widehat{X}_{t}[\varphi] Y_{t}[\varphi]=n_{t}^{\varphi}+\int_{0}^{t}\left(\alpha_{s}^{\varphi}+\beta_{s}^{\varphi}\right) \mathrm{d} s+\int_{0}^{t} Y_{s}[\varphi]\left(A \widehat{X}_{s}\right)[\varphi] \mathrm{d} s
$$

where $n_{t}^{\varphi}$ is a real valued square integrable $\left\{\mathcal{G}_{t}\right\}$-martingale, $\alpha_{t}^{\varphi}=\widehat{X}_{t}[\varphi] \widehat{H\left(X_{s}\right)}[\varphi]$ and $\beta_{t}^{\varphi}=Q\left(Z^{*}(t) \varphi, \varphi\right), 0 \leq t \leq T$. Comparing (5.2) and (5.6), and according to the uniqueness of canonical decomposition of semimartingales, we get

$$
\int_{0}^{t}\left(\widehat{a}_{s}^{\varphi}+\widehat{b}_{s}^{\varphi}\right) \mathrm{d} s=\int_{0}^{t}\left(\alpha_{s}^{\varphi}+\beta_{s}^{\varphi}\right) \mathrm{d} s, \quad 0 \leq t \leq T,
$$

or $Q\left(Z^{*}(t) \varphi, \varphi\right)=\widehat{a}_{t}^{\varphi}+\widehat{b}_{t}^{\varphi}-\widehat{X}_{t}[\varphi] \widehat{H\left(X_{s}\right)}[\varphi], 0 \leq t \leq T$. Noting that

$$
a_{t}^{\varphi}=X_{t}[\varphi] Q(h(t), \varphi)=Q((X \otimes h)(t) \varphi, \varphi), \quad 0 \leq t \leq T
$$

and

$$
\widehat{X}_{t}[\varphi] \widehat{H\left(X_{s}\right)}[\varphi]=\widehat{X}_{t}[\varphi] Q(\widehat{h}(t), \varphi)=Q((\widehat{X} \otimes \widehat{h})(t) \varphi, \varphi), \quad 0 \leq t \leq T
$$

we have

$$
Q\left(Z^{*}(t) \varphi, \varphi\right)=Q\left(\left[(\widehat{X \otimes h})(t)+\widehat{F}^{*}(t)-(\widehat{X} \otimes \widehat{h})(t)\right] \varphi, \varphi\right), \quad 0 \leq t \leq T .
$$

Hence, by Lemma 5.1, we get

$$
Z(t)=(\widehat{h \otimes X})(t)+\widehat{F}(t)-(\widehat{h} \otimes \widehat{X})(t), \quad 0 \leq t \leq T
$$

since $Q$ is strictly positive and $\varphi \in \Phi$ is arbitrary. Substituting $Z(\cdot)$ to equation (5.4) we obtain the equation (5.1). The proof is completed. 


\section{References}

[1] D. Ding, Stochastic integration for operator valued processes on dual of nuclear Fréchet space and its application, Applied Probability and Statistics, Vol.7(1991), pp.381-389.

[2] D. Ding, Stochastic evolution equations in duals of nuclear Fréchet Spaces, in Nonlinear Evolution Equations and Their Applications, eds by Ta-tsien Li, Long-wei Lin and J.F. Rodrigues, 1999, World Scientific.

[3] M.Fujisaki, G. Kallianpur and H. Kunita, Stochastic differential equations for the nonlinear filtering problem, Osaka J. Math. 9, pp.19-40, 1972.

[4] N.Ikeda and S.Watanabe, Stochastic Differential Equations and Diffusion Processes, North-Holland Publishing Company 1981.

[5] H. Korezioglu, Nonlinear filtering equation for Hilbert space valued process, in R.S. Bucy and J.M.F. Moura eds. Nonlinear Stochastic Problem, Reidel Pub. Co., 1983.

[6] H. Korezioglu and C. Martias, Martingale representation and nonlinear filtering equation for distribution valued processes, Lecture Note in Control and Information Sciences 61, springer-Verlag, 1984.

[7] H. Korezioglu and C. Martias, Stochastic integrations for operator valued processes on Hilbert spaces and on nuclear spaces, Stochastics, Vol.24, pp.171-219, 1988.

[8] M. Metivier, Semimartingals, a Course on Stochastic Processes, Walter de Gruyter, 1982.

[9] J.Y. Ouvrard, Martingale projection and linear filtering in Hilbert spaces, SIAM J. Control and Optimzation 16(1978), pp.912-937.

[10] H.H. Sharfer, Topological Vector Spaces, Springer-Verlag, 1971.

[11] A.S. Ustunel, Stochastic integration on nuclear space and its applications, Ann.Inst.Henri. Poincare, Section B, Vol.18(1982), pp.165-200.

Received: July 17, 2006 